\title{
Using the PEPPA Framework to Develop and Implement a Nurse Practitioner Role Within Canada's National Ballet School
}

\author{
Erin Ziegler, PhD, NP-PHC ${ }^{1}$ \\ eziegler@ryerson.ca \\ Sophia Kim, MPH ${ }^{2}$ \\ sophia.kim@nbs-enb.ca \\ Rachel J. Bar, $\mathrm{PhD}^{2}$ \\ rbar@nbs-enb.ca \\ 1. Daphne Cockwell School of Nursing, Ryerson University, Toronto, Ontario, Canada \\ 2. Canada's National Ballet School, Toronto, Ontario, Canada
}

\begin{abstract}
Aim: To outline the successful development and implementation of a nurse practitioner role within a professional ballet school.

Background: Nurse practitioners are well integrated into primary and acute care in Ontario, yet the role within schools and private athletic institutions is not well documented. Canada's National Ballet School is a professional ballet school with a combination of day students and those living in residences. Students complete both dance training and academics at the School. The physical and mental health of students was identified as a key priority by the school, leading to the development of an integrated health and wellness program. To facilitate more timely access to healthcare and provide an opportunity for collaboration and consultation within the school, a plan to implement a nurse practitioner role into the school was developed.

Methods: In order to develop and implement the role of the nurse practitioner within the institution, the participatory, evidence-based, patient-focused process for advanced practice nursing role development, implementation, and evaluation (PEPPA) framework was used. The first seven steps of the PEPPA framework were applied in this project.

Findings: The PEPPA framework allowed for us to identify key barriers and facilitators for the role implementation and successfully implement the nurse practitioner role. While the initial plan was for a slower implementation, the COVID-19 pandemic highlighted the need for a nurse practitioner in the institution more urgently.

Conclusion: The PEPPA framework provided us with an organized process for developing and implementing the nurse practitioner role at Canada's National Ballet School.
\end{abstract}

Key Words: Nurse Practitioners; Advanced Practice Nursing; School; Dancing; Nurse’s Role

\section{Introduction}

Canada's National Ballet School (NBS) is recognized internationally for its professional ballet and academic program. The Olympic caliber training program for talented young dancers aged 11 to 19 is highly competitive. Acceptance into the school is by audition only and reacceptance is required for returning students each year. Students at NBS complete all their dance training and Ontario Ministry of Education academic curriculum onsite. In a typical year, 
approximately $60 \%$ of students live in residence, while the remaining $40 \%$ commute to school as day students. Youth attending boarding schools experience a separation from their home environment. While this transition for most can promote personal growth, for some it can be disruptive (Gottlieb, 2001). Youth who live away from home may not be aware of, or be able to independently recognize their emotional difficulties or physical illnesses, therefore relying more on the support from adults at the school (Pavletic et al., 2016). While most healthcare issues affecting youth relate to acute episodic illness or injury and preventative care, elite dancers may experience additional healthcare issues. Dance related injuries, malnutrition, disordered eating, body dissatisfaction are common primary care issues identified for dancers (Alimena et al, 2016; Russell, 2013). Furthermore, injury and the duration of recovery, plus the frequency of injury have been linked to increased stress, anxiety, depression, and fatigue in ballet dancers (Alimena et al., 2016; Jacobs et al., 2012; Noh, 2005).

Until the recent developments described in this paper, NBS' health and wellness services for students included daily access to onsite physiotherapy, and offsite access to a podiatrist, sport physician, nutritionist, and mental health professionals. A family physician came onsite once a week to see students living in residence needing attention.

Improving access to primary care services can positively affect dancers' health and performance (Russell, 2013). A study by Nygren et al., (2013) demonstrated that school-based health promotion interventions need to be focused on the students' individual needs.

Furthermore, students benefit from the support provided by school nurses (Nygren et al., 2013).

\section{Nurse Practitioners in Schools}

Nurse practitioners (NPs) are advanced practice nurses with extended scope of practice. In Canada, this extended scope of practice allows NPs to independently diagnose and treat, order diagnostic tests, refer to specialists, perform procedures and, if working in a hospital, admit and discharge patients (Spence et al., 2015). Most NPs in Canada practice in primary care settings (Alden-Bugden, 2019), and research has demonstrated high levels of patient safety, efficacy, and cost-effectiveness with the NP role (Stanik-Hutt et al., 2013; Martin-Misener et al., 2015). While the role of NPs has been well documented in primary and acute care settings, the role in elementary and high schools is a novel approach. For this reason, it is not surprising that a search of the literature yielded minimal discussion of NPs in elementary and high schools.

Historically, in schools the role of the NP has involved improving health habits of students and providing health education (Weber, 2006). In the elementary school setting NPs have been involved in the Whole School, Whole Community, Whole Child model which aims to improve the overall health of students. The model includes health education, physical education, health services, nutrition services, health promotion for staff, counseling and psychological services, healthy school environment, and parent/community involvement (Center for Disease Control and Prevention, 2014; Weber, 2006). In high schools, NPs in school-based health centers provide comprehensive health and mental care services. Access to these services in school settings eliminates barriers adolescents encounter seeking care (Daley \& Polifroni, 2018; Daley, Polifroni \& Sadler, 2019). Students have expressed comfort and the ability to establish trusting relationships with NPs in these school-based health centers (Daley, Polifroni \& Sadler, 2019). 
Furthermore, in university health centers NPs provide direct patient care, health education, health promotion, and disease prevention activities (Hicks \& Dinkel, 2016).

The role of registered nurses (RNs) in public schools has been well documented in the literature, highlighting the important link between health and education. No literature was found discussing the nursing role in private or boarding schools. The role of school nurses in Canada is not well documented, the role is prominent in other countries. In the United States, the National Association of School Nurses (2018) and the American Academy of Pediatrics (2016) have advocated for school nurses to be in every school, every day of the school year. From an organizational perspective, having a school nurse has been shown to be cost effective, reducing use of community health services, decreasing the time that parents need to be away from work, and reducing the time teachers spend addressing health issues (Biag et al., 2018; Dolatowski et al., 2016; Lineberry et al., 2018). Population health promotion, including screening, vaccinations, infection control, injury prevention and health promotion is a foundation of school nursing (Bergren, 2017). A study by Kocoglu and Emiroglu (2017) found that nursing interventions resulted in statistically significant improvement in students' quality of life and physical, mental and behavioural health scores. School nurses promote health in the school and community.

\section{PEPPA Framework}

Developed by Bryant-Lukosius \& DiCenso (2004), the Participatory, Evidence-based, Patient-centered process for Advanced practice nurse (APN) role development, implementation, and evaluation (PEPPA) framework is a tool for implementation and utilization of the APN role. The framework addresses recommendations to address challenges with implementation using an evidence-based process to (i) support the need, identify goals, and clearly define the role, (ii) support the development of APN practice, (iii) promote APNs to work at full scope of practice, (iv) support role development within the healthcare setting and system, and (v) rigorously evaluate the role (Bryant-Lukosius \& DiCenso, 2004). The PEPPA framework has effectively been used in practice settings to assist in the development, implementation, and evaluation of APN roles (Boyko, Carter \& Bryant-Lukosius, 2016).

The PEPPA Framework defines 9 key steps for effectively assessing the need for an advanced practice nurse and for developing and implementing this role within the practice setting (see Figure 1). The purpose of this paper is to describe the implementation of the NP role within NBS using the PEPPA framework. The first seven steps of the framework will be described. 
Figure 1: PEPPA Framework.

Used with permission from Bryant-Lukosius \& DiCenso (2004)

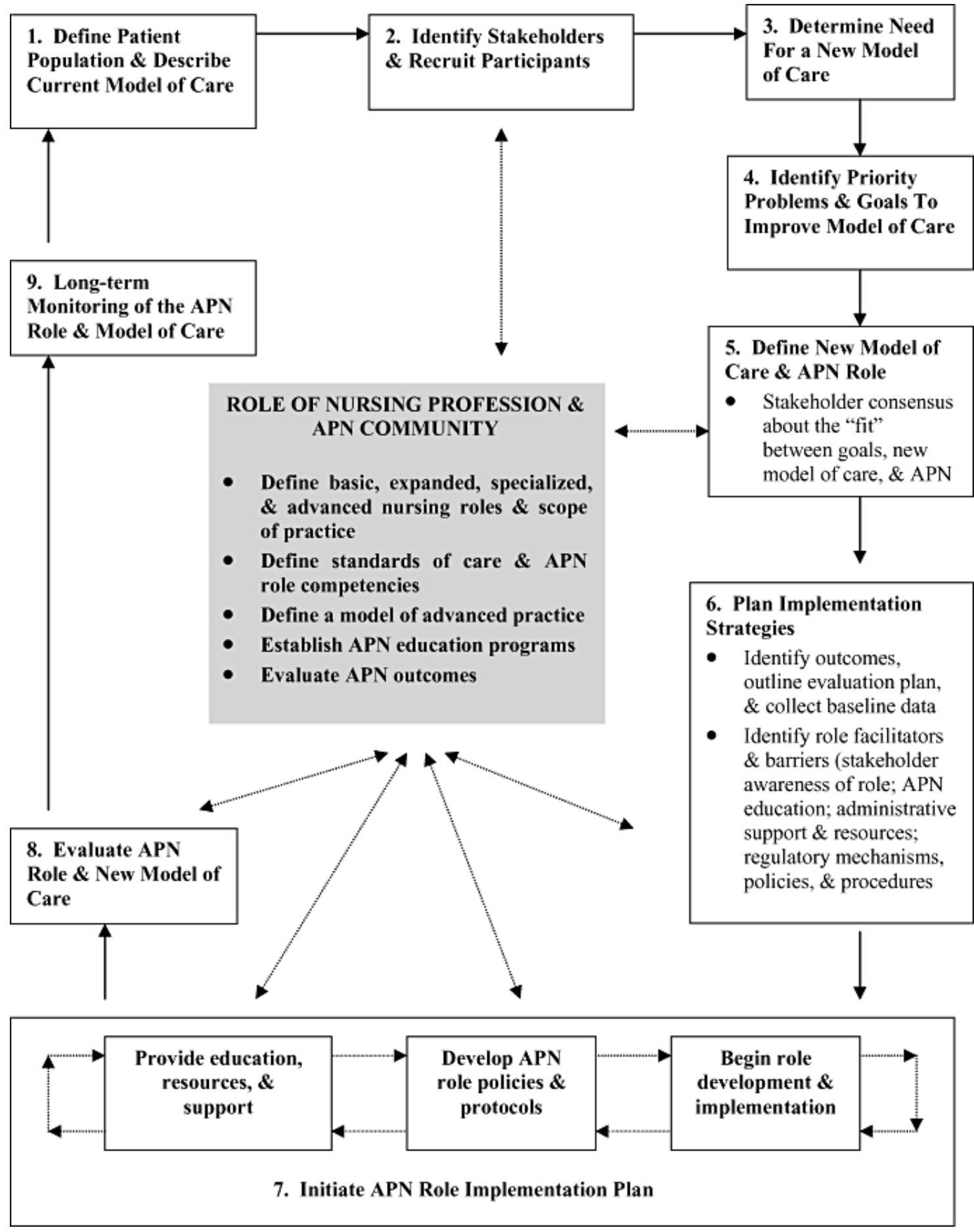




\section{Step 1: Define Patient Population and Describe Current Model of Care}

The population of NBS' professional ballet/academic program includes children and youth between the age of 11 and 19 (academic grades 6-12 and post-secondary). Given the unique NBS population of young elite athletes, most living away from home, NBS' model of healthcare included a fee-for-service physician who visited weekly from their nearby private practice to check the health of students living in residence. NBS has onsite physiotherapy and works with several off-site healthcare professionals, including a podiatrist and sport physicians for injuries and mental health professionals, such as psychologists and social workers, to support student mental health. When service costs are not covered by student insurance, NBS generally covers student healthcare costs. Between all these services, stakeholders acknowledged that supports were not necessarily optimally integrated, creating gaps and discontinuity of care. Students' route to accessing healthcare also lacked any centralized process and would be arranged by residence, academic, or artistic staff. Although this provided multiple accessible routes to care for students, it made it difficult to understand and integrate student care needs across the school. Further, with each specialty working separately from the others, this model limited the opportunity to support integrated prevention initiatives thus also limiting students' opportunity to maximize their potential as dancers.

\section{Step 2: Identify Stakeholders and Recruit Participants}

Over the years, NBS has moved toward an evidence-informed model of health and wellness for its students. This approach has been directed by the School's leadership, including the Artistic Director, Executive Director, and NBS Board Members. The key stakeholders involved in the decision to introduce a full-time NP at NBS included these stakeholders as well as leadership within the artistic, residence, and academic faculty. Additional advocates for the introduction of the role included NBS' Director of Research and Health as well as parents of students. Several discussions amongst all of these stakeholders occurred before the decision to move forward with this role. These discussions were initiated by the Artistic Director and Executive Director with the various stakeholder groups.

\section{Step 3. Determine Need for New Model of Care}

As a boarding school, NBS is responsible for arranging the primary care of its students living in residence. This responsibility is overseen by the School's Artistic Director and CEO, but was typically managed by staff who worked in the residence who would arrange for primary care as needed. NBS also endeavors to oversee the unique care needs of all of its students as they relate to training as elite athletes/dancers. The students at the school need access to care both for their needs as growing children/youth, but also for the specific needs that come with being an elite athlete/dancer (e.g., foot care and sports medicine).

To support more efficient and effective care and training goals, NBS made the decision to move toward an integrated model of care in early 2020. Growing evidence in both healthcare (Peña-Sánchez et al., 2017; Buchman et al., 2018; Gordon et al., 2020) and athlete care (Dijkstra et al., 2014; DiFrancisco-Donoghue et al., 2019) suggest that an integrated model may lead to better outcomes. Consideration of this literature led to the formation of the Artistic Health 
Department, which oversees the integration of student health and wellness care and maximizing student potential as elite athletes/dancers. Health and wellness care for students can include acute and chronic care management, sexual health, mental health and social support. Plans to evolve the health program and development of the Artistic Health Department were discussed prior to the COVID 19 pandemic, but the department was not officially established until September 2020. The department includes both healthcare professionals (e.g., physiotherapist and nurse practitioner) and trainers (e.g., conditioning instructor, yoga instructor) to support students' dance training.

The addition of the NP to this department was thought to support the integration of students' primary care needs into the overall care/training of students, thus enhancing consistency and effectiveness of care for the students. It is noteworthy however that the specific need for the NP was not directly identified until the pandemic emerged. After the initial lockdown caused by the pandemic, the School began to plan for reopening which quickly brought the need for the NP into focus. Reopening the School after the province closed all schools in spring 2020 would not only require planning but ongoing assessment and decision making. The NP would not only be able to assess and guide students in issues related to the pandemic, but their knowledge and experience as an NP would also help inform the school's policies and management of the situation. While it is likely the School would have recognized the need to invest in a full-time NP even without the pandemic, the situation was clearly a catalyst. Recognizing the immediate need as well as the potential of an NP to support the vision of the Artistic Health Department, all stakeholders agreed that creating the NP role would support the school's needs during the pandemic and beyond.

\section{Step 4. Identify Priority Problems and Goals}

The priority problem identified by key stakeholders was the lack of access to healthcare within a timely manner for students. This problem was exacerbated by COVID 19 but. in fact, was a pre-existing concern. Students living in the residence away from home had no regular access to a primary care provider, often needing to wait to see the visiting physician or going to a walk-in clinic. Furthermore, there was the potential for delays in diagnosis and treatment if students waited until they went home on break to see their regular primary care provider. Lack of coordinated primary care services was identified as a gap, as current services focused on acute and episodic illness and injuries. Primary preventative care such as vaccinations, infection control and integrated healthcare services was missing.

The main goal was to develop an integrated healthcare program which maximizes the potential for students to access healthcare services for routine, acute and preventative care. Providing this service on-site increases students' access to these services and reduces the resource intensive support needed from staff to arrange and accompany students off-site for medical appointments.

\section{Step 5. Define New Model of Care and APN Role}

Recognizing the potential to support student training, health, and wellbeing more optimally, NBS began to consider what a more integrated model of health and well-being 
services could look like for students. The new model of care would situate itself within a new department, the Artistic Health Department. Experts from various fields related to training dancers (e.g., conditioning instructor, yoga instructor) and healthcare professionals (e.g., physiotherapist and nurse practitioner) would not just work with the students as they had done previously, but now as a team within the department they would work together to support students. The head of the department would also meet regularly with artistic, academic and residence management to stay informed about school matters and each team member would also be encouraged to engage in different aspects of the school more directly. For example, the physiotherapist would be encouraged to block time off each week to view the students training in the dance studios to better inform prevention and treatment. While it was clear that the unique context of NBS would require its own unique approach to integration, as a model, it would share similarities with other integrated models of care for athletes (Dijkstra et al., 2014; Sudano et al., 2017).

The role of an NP is seen as an ideal fit within the newly developed Artistic Health Department. The autonomy of the NP scope of practice allows for them to be key members of the healthcare team. In Ontario, NPs are authorized to diagnose, prescribe medications and treatments and to order and interpret diagnostic tests (College of Nurses of Ontario, 2019). To aid in the implementation of the NP role, NBS engaged the assistance of an experienced primary care NP to help develop the job description and define the role of the NP in the newly developed Artistic Health Department. The new model of care will focus on the implementation of an NP. The role of the NP will be to provide regular on-site access to healthcare, streamline and coordination of services and provide students with a more private and discreet approach to care as they can access care independently and no longer rely on staff to assist them in arranging care. Furthermore, as a private school, NBS has identified the healthcare of their dancers as a top priority.

Despite the numerous advantages identified there were also some key disadvantages identified. One current limitation is the current barrier in scope of practice for NPs to independently order MRIs and CT scans (College of Nurses of Ontario, 2019). While this legislative change to allow ordering these tests is expected to come shortly, NPs currently are still required to consult with a physician if a patient requires an MRI or CT scan.

Another potential challenge for this role is the context. As an arts institution, the culture of practice may vary greatly from the healthcare settings an NP would be most familiar. Understanding of the NP role and clarity of the role is key to integration within the institution. Lack of role clarity has been identified as a barrier to successful NP role implementation (Carter et al., 2010; Donald et al., 2010; Sangster-Gormley et al., 2011). Healthcare professionals are accountable to their colleges and must adhere to strict regulations when it comes to who and what they can treat, how it is reported, and how information is shared. These requirements would be very different from the professionals working in an arts institution or even a school.

Non-healthcare professionals at NBS may have very different, or no regulating body for their profession. This may present as a challenge when care is aiming to be integrated, especially when a student's situation needs to be discussed among varying staff. For example, if a student's health issue required modification to their dance training, the NP would need the student's 
consent to discuss the issue with their dance teacher. This highlights the need for clear disclosure consent protocols to be created with the NP role.

\section{Step 6. Plan Implementation and Strategies}

NBS has set both short and long-term goals associated with the creation of the NP role. In the short-term, keeping the school safe and ideally open for training during the pandemic is critical. The NP will help ensure students are training safely by providing onsite healthcare service for students and inform school policy related to infection control and the pandemic. In both the short and long-term, the NP role will improve access to primary care for students. The overarching long-term goal of the NP role would be to support an integrated model of care for the students. As outlined earlier, an integrated care model is needed to optimize students' development as future professional dancers. A NP who understands the needs of a dance trainee while providing healthcare interventions is an imperative next step to maximize students' potential and wellbeing at NBS.

Role facilitators within NBS include its advocates, such as the Director of Research and Health, who will be able to support the integration of the NP role into the school. The School's commitment to evidence informed practice will also support the facilitation of this role, and inevitably the COVID-19 pandemic has highlighted critical gaps in student healthcare and has facilitated the prioritization of the NP role at NBS.

Organizational barriers to NP role implementation in the literature have included lack of administrative support, organizational cultures, lack of job descriptions and conflicting role expectations (Sangster-Gormley et al., 2011). Potential role barriers identified may include an organization culture drastically different from a typical healthcare setting, space on campus for the new role, lack of access to healthcare supports such as electronic medical records, subscription-based clinical practice guidelines, and research journals.

\section{NP mentor}

To support the implementation of the role, a mentorship program was developed. The NP will be the first and only primary care provider at NBS, therefore, to ensure the opportunity for collaboration and support a formal mentorship will be implemented with a primary care NP who has experience in NP role development. Formal mentorship has been identified as a key facilitator due to the complexity of NP role implementation (Sangster-Gormley et al., 2011).

\section{Administrative Support and Resources}

Both the manager of the Artistic Health Department (i.e., Director, Research and Health) and the department's coordinator will be available to support the introduction of the NP role to students and staff. Because this is a new position unlike any previous role, the NP will be able to develop policies and procedures for the specific nature of the role with the support of these colleagues as well as the external NP mentor. 


\section{Step 7: Initiate APN Role Implementation Plan}

The NP role was implemented at NBS in September 2020. While a slower integration in which policies and procedures could be established before students began a new academic year may have been ideal, due to the COVID-19 pandemic this was not possible. The implementation plan needed to be fast tracked as there was an urgency for healthcare services to be available for students who were not feeling well. Therefore, there was limited time to prepare for the role implementation, both for the organization and the NP.

At the time of this publication, several policies and procedures had already been developed in relation to the NP role and several were still undergoing development. For example, an appointment booking system, electronic medical record, and communication policy were already established. The NP has also begun to lead sex education workshops with students previously outsourced or led by academic teachers. Much of the NP's time since implementation has also had to focus on supporting COVID-19 policies and procedures. As the NP in the role learns more about the school's students and their specific needs, the role will no doubt continue to evolve.

\section{Discussion}

The PEPPA framework has been used internationally to introduce and implement the role of APNs in various healthcare systems (Bryant-Lukosius et al., 2016), however to our knowledge this is the first time it has been used in a private, dance and academic institute. The framework provided a systematic and structured process to decision-making in collaboration with stakeholders, to develop a role and model of care that meets the needs of NBS (Bryant-Lukosius \& DiCenso, 2004). Having a structured process was important when developing a new NP role within an existing institution and provided a guide for introducing the new role as well as understanding the factors involved in the implementation. Due to the COVID-19 pandemic, the role was implemented quickly to address urgent healthcare needs of the students and the School. Ideally, the implementation would have been rolled out slowly to allow for easier integration, both for the staff and students at NBS and the NP starting in the role. Despite the challenges of the rapid implementation and the COVID-19 pandemic, the new model of care and the NP were successfully initiated at NBS.

This paper discussed the first seven steps of the PEPPA framework. The final two steps involve evaluating and long-term monitoring of the role and model of care. While the role has recently been implemented, we outline out plans for step eight and nine.

\section{Step 8: Evaluation Methods}

To understand the implementation experience and evaluate its success, several tools will be developed including surveys and scripted interviews. Specifically, these tools will evaluate (1) student experiences of the role, including their experience accessing care and understanding if/how the service has helped them, (2) administration experiences, including their perspective on how the role has supported NBS policies and processes, (3) staff experiences, including the impact of the NP role on their job, (4) parent experiences, including their experience of the role 
at NBS, and (5) the experience of the NP implementing the role. Formal evaluation will begin in Fall 2021, one year after the implementation of the NP role.

\section{Step 9: Long Term Monitoring of the Role and Model of Care}

Long-term monitoring of the NP role will include annual formal evaluation through student surveys and ongoing conversations with the NP in the role. In addition to overall integration and effectiveness of the role, the evolution of the role once the COVID-19 pandemic has abated will be considered in the longer-term evaluation process. Furthermore, long-term monitoring can be used to direct any changes that need to be made to the role or model of care.

\section{Conclusion}

The value of having an integrated model of care at NBS has been acknowledged by key stakeholders and implementing a NP on site is a key component of the new model. Using the PEPPA framework helped us to ensure successful implementation, despite the accelerated process due to the needs from the current pandemic. The PEPPA framework provided us with a structured and systematic approach to successfully integrate the role of the NP into NBS. We are hopeful that long-term evaluations and monitoring of the new model of care will demonstrate that the role of the NP was imperative to NBS and potentially serve as a model for the implementation of the role in other professional ballet schools or similar elite athlete training institutions.

\section{References}

Alden-Bugden, D. (2019). The role and scope of the NP in Canada. The Nurse Practitioner, 44(9), 8-9. https://doi.org/10.1097/01.NPR.0000577972.5019.34

Alimena, S., Air, M.E., Gribbin, C. \& Manejias, E. (2016). Utilization of routine primary care services among dancers. Journal of Dance Medicine \& Science, 20(3), 95-102. https://doi.org/10.12678/1089-313X.20.3.95

American Academy of Pediatrics. (2016). Role of the school nurse in providing school health services. Pediatrics, 137(6):e20160852. https://doi.org/10.1542/peds.2016-0852

Bergren, M.D. (2017). School nursing and population health: Past, present, and future. The Online Journal of Issues in Nursing, 22(3). https://doi.org/10.3912/OJINVol22No03Man03

Biag, M., Srivastava, A., Landau, M., \& Rodriguez, E. (2015). Teachers' perceptions of full and part-time nurses at school. Journal of School Nursing, 31(3), 83-195. https://doi.org/10.1177/1059840514561863 
Nurse Practitioner Open Journal

Vol 1. Issue 1 DOI 10.28984/npoj.v1i1.341

Boyko, J.A., Carter, N. \& Bryant-Lukosius, D. (2016). Assessing the spread and uptake of a framework for introducing and evaluating advanced practice nursing roles. Worldviews of Evidence-Based Nursing, 134, 277-584. https://doi.org/10.1111/wvn.12160

Bryant-Lukosius, D. \& DiCenso, A. (2004). A framework for the introduction and evaluation of advanced practice nursing roles. Journal of Advanced Nursing, 48, 530-540.

DOI: $10.1111 / \mathrm{j} .1365-2648.2004 .03235 . \mathrm{x}$

Bryant-Lukosius, D., Spichiger, E., Martin, J., Stoll, H., Kellerhals, S.D., Fliedner, M., Grossmann, F., Henry, M., Herrmann, L., Koller, A., Schwendimann, R., Ulrich, A., Weibel, L., Callens, B. and De Geest, S. (2016). Framework for evaluating the impact of advanced practice nursing roles. Journal of Nursing Scholarship, 48(2), 201-209. https://doi.org/10.1111/jnu.12199

Buchman, S., Evans, J. M., MacKinnon, M., Gradin, S., \& Wright, F. C. (2018). Bridging silos: Delivering integrated care to patients with cancer in Ontario, Canada. Journal of the Psychological, Social and Behavioural Dimensions of Cancer. 27(12):2673-2676. https://doi.org/10.1002/pon.4858

Carter, N., Martin-Misener, R., Kilpatrick, K., Kaasalainen, S., Donald, F., Bryant-Lukosius, D., Harbman, P., Bourgeault, I. \& DiCenso, A. (2010). The role of nursing leadership in integrating clinical nurse specialists and nurse practitioners in healthcare delivery in Canada. Canadian Journal of Nursing Leadership, 23(special issue), 167-185. https://doi:10.12927/cjnl.2010.22274.

Center for Disease Control and Prevention (2014). Whole school, whole community, whole child: A collaborative approach to learning and health. Retrieved from https://www.cdc.gov/healthyschools/wscc/wsccmodel_update_508tagged.pdf

College of Nurses of Ontario (2019). Practice standard: Nurse practitioner. Retrieved from https://www.cno.org/globalassets/docs/prac/41038_strdrnec.pdf

Daley, A.M., \& Polifroni, E.C. (2018). "Contraceptive care for adolescents in school-based health centers is essential!" The lived-experience of nurse practitioners. Journal of School Nursing, 34(5), 367-379. https://doi.org/10.1177/1059840517709503

Daley, A.M., Polifroni, E.C. \& Sadler, L.S. (2019). The essential elements of adolescent-friendly care in school-based health centers: A mixed methods study of the perspectives of nurse practitioners and adolescents. Journal of Pediatric Nursing, 47, 7-17. https://doi:10.1016/j.pedn.2019.03.005

DiFrancisco-Donoghue, J., Balentine, J., Schmidt, G., \& Zwibel, H. (2019). Managing the health of the eSport athlete: An integrated health management model. BMJ Open Sport \& Exercise Medicine. 5:e00467. https://doi.org/10.1136/bmjsem-2018-000467 
Nurse Practitioner Open Journal

Vol 1. Issue 1 DOI 10.28984/npoj.v1i1.341

Dijkstra, H. P., Pollock, N., Chakraverty, R., \& Alonso, J. M. (2014). Managing the health of the elite athlete: A new integrated performance health management and coaching model. British Journal of Sports Medicine, 48(7), 523-531. https://doi.org/10.1136/bjsports2013-093222

Dolatowski, R., Endsley, P., Hiltz, C., Johansen, A., Maughan, E., Minchella, L. \& Trefry, S. (2015). School nurse workload - staffing for safe care: Position statement. NASN School Nurse. 2015 Sep;30(5):290-3. https://doi.org/10.1177/1942602X15594143

Donald, F., Bryant-Lukosius, D., Martin-Misener, R., Kaasalainen, S., Kilpatrick, K., Carter, N., Harbman, P., Bourgeault, I \& DiCenso, A. (2010). Clinical nurse specialists and nurse practitioners: Title confusion and lack of role clarity. Canadian Journal of Nursing Leadership, 23(special issue), 189-201. https://doi: 10.12927/cjnl.2010.22276

Gordon, K., Gray, C. S., Dainty, K. N., DeLacy, J., Ware, P., \& Seto, E. (2020). Exploring an innovative care model and telemonitoring for the management of patients with complex chronic needs: Qualitative description study. JMIR Nursing. 3(1):e15691.https://doi.org/10.2196/15691

Gottlieb, R. M. (2001). Consultation and therapy in the boarding high school setting. Child and Adolescent Psychiatric Clinics of North America, 10, 139-149.

DOI: $10.1016 / \mathrm{s} 1056-4993(18) 30079-8$

Hicks, R.W. \& Dinke, S. (2016). The hidden gems in student health centers. Journal of the American Association of Nurse Practitioners, 28(4), 178-80. https://doi: 10.1002/23276924.12364.

Jacobs, C.L., Hincapie, C.A., \& Cassidy, J.D. (2012). Musculoskeletal injuries and pain in dancers: A systematic review update. Journal of Dance Medicine \& Science, 16(2):74-84. doi: 10.1016/j.apmr.2008.02.020

Kocoglu, D. \& Emiroglu, O.N. (2017). The impact of comprehensive school nursing services on students' academic performance. Journal of Caring Sciences, 6(1), 5-17. https://doi.org/10.15171/jcs.2017.002

Lineberry, M., Whitney, E. \& Noland, M. (2018). The role of school nurses, challenges, and reactions to delegation legislation: A qualitative approach. Journal of School Nursing, 34(3), 222-231. https://doi.org/10.1177/1059840517741526

Martin-Misener, R., Harbman, P., Donald, F., Reid, K., Kilpatrick, K., Carter, N., BryantLukosius, D., Kaasalainen, S., Marshall, DA., Charbonneau-Smith, R. \& DiCenso, A. (2015) Cost-effectiveness of nurse practitioners in primary and specialized ambulatory care: Systematic review. BMJ Open. 5(6):e007167. https://doi.org/10.1136/bmjopen2014-007167 
Nurse Practitioner Open Journal

Vol 1. Issue 1 DOI 10.28984/npoj.v1i1.341

National Association of School Nurses (2018). Strategic plan: 2020-2030. Retrieved from https://www.nasn.org/about-nasn/about

Noh, Y. (2005). Psychosocial factors and ballet injuries. International Journal of Sport Exercise and Psychology, 3(1), 7-25. https://doi.org/10.1080/1612197X.2005.9671759

Nygren, K., Bergstom, E., Janlert, U., \& Nygren, L. (2013). Adolescent self-reported health in relation to school factors: A multilevel analysis. The Journal of School Nursing, 30, 114122. https://doi.org/10.1177/1059840511389709

Pavletic, A.C., Dukes, T., Gardine Greene, J., Taylor, J., \& Gilpin, L.B. (2016). Health services in boarding school: An oasis of care, counseling, and comfort. The Journal of School Nursing, 32(5), 304-314. https://doi.org/10.1177/1059840516649234

Peña-Sánchez, J. N., Lix, L. M., Teare, G. F., Li, W., Fowler, S. A., \& Jones, J. L. (2017). Impact of an integrated model of care on outcomes of patients with inflammatory bowel diseases: Evidence from a population-based study. Journal of Crohn's and Colitis, 11(12), 1471-1479. https://doi.org/10.1093/ecco-jcc/jjx106

Russell, J.A. (2013). Preventing dance injuries: Current perspectives. Open Access Journal of Sports Medicine, 30(4), 199-210. https://doi.org/10.2147/OAJSM.S36529

Sangster-Gromley, E., Martin-Misener, R., Downe-Wambold, Bt \& DiCenso, A. (2011). Factors affecting nurse practitioner role implementation in Canadian practice settings: An integrative review. Journal of Advanced Nursing, 67(6), 1178-1190. https://doi.org/ 10.1111/j.1365-2648.2010.05571.

Spence, L., Agnew, T. \& Fahey-Walsh, J. (2015). A Pan-Canadian environmental scan of the scope of practice of nurse practitioners. Retrieved from http://npao.org/pdf/A_PanCanadian_Environmental_Scan_of_NPs_2015.pdf

Stanik-Hutt, J., Newhouse, R.P., White, K.M., Johantgen, M., Bass, E.B., Zangaro, G., Wilson, R., Fountain, L., Steinwachs, D.M., Heindel, L. \& Weiner, J.P. (2013). The quality and effectiveness of care provided by nurse practitioners. The Journal for Nurse Practitioners, 9(8), 492-500. https://doi.org/10.1016/j.nurpra.2013.07.004

Sudano, L., Collins, G., \& Miles, C. (2017). Reducing barriers to mental health care for studentathletes: An integrated care model. Families, Systems \& Health. 35(1):77-84. https://doi.org/10.1037/fsh0000242

Weber, S. (2006). School health programs: A starring role for school nurse practitioners! Journal of the American Academy of Nurse Practitioners, 18(11), 510-511. https://doi.org/10.1111/j.1745-7599.2006.00170.x 